\title{
MiR-593 mediates curcumin-induced radiosensitization of nasopharyngeal carcinoma cells via MDR1
}

\author{
HAONING FAN ${ }^{1 *}$, MENG SHAO $^{1 *}$, SHAOHUI HUANG $^{1 *}$, YING LIU $^{2}$, JIE LIU $^{2}$, \\ ZHIYUAN WANG ${ }^{2}$, JIANXIN DIAO ${ }^{1}$, YUANLIANG LIU ${ }^{1}$, LI TONG ${ }^{1}$ and QIN FAN ${ }^{1}$ \\ ${ }^{1}$ Department of Molecular Biology, School of Traditional Chinese Medicine; \\ ${ }^{2}$ Department of Radiotherapy, Nanfang Hospital, Southern Medical University, Guangzhou, Guangdong 510515, P.R. China
}

Received February 17, 2015; Accepted March 8, 2016

DOI: $10.3892 / \mathrm{ol} .2016 .4438$

\begin{abstract}
Curcumin (Cur) exhibits radiosensitization effects to a variety of malignant tumors. The present study investigates the radiosensitizing effect of Cur on nasopharyngeal carcinoma (NPC) cells and whether its mechanism is associated with microRNA-593 (miR-593) and multidrug resistance gene 1 (MDR1). A clonogenic assay was performed to measure the radiosensitizing effect. The expression of miR-593 and MDR1 was analyzed by quantitative polymerase chain reaction (qPCR) or western blot assay. A transplanted tumor model was established to identify the radiosensitizing effect in vivo. A luciferase-based reporter was constructed to evaluate the effect of direct binding of miR-593 to the putative target site on the 3' UTR of MDR1. The clonogenic assay showed that Cur enhanced the radiosensitivity of cells. Cur $(100 \mathrm{mg} / \mathrm{kg})$ combined with 4 Gy irradiation inhibited the growth of a transplanted tumor model in vivo, resulting in the higher inhibition ratio compared with the radiotherapy-alone group. These results demonstrated that $\mathrm{Cur}$ had a radiosensitizing effect on NPC cells in vivo and in vitro; Cur-mediated upregulation of miR-593 resulted in reduced MDR1 expression, which may promote radiosensitivity of NPC cells.
\end{abstract}

\section{Introduction}

Nasopharyngeal carcinoma (NPC), a type of malignant head-and-neck cancer, is a geographical predilection in Southeast Asia, particularly in the Southern provinces of China with

Correspondence to: Professor Qin Fan or Professor Li Tong, Department of Molecular Biology, School of Traditional Chinese Medicine, Southern Medical University, 1,838 Guangzhou Avenue North, Guangzhou, Guangdong 510515, P.R. China

E-mail: fqin@163.com

E-mail: zyxy2@fimmu.com

*Contributed equally

Key words: radiosensitization, curcumin, multidrug resistance gene 1, microRNA-593, nasopharyngeal carcinoma an estimated incidence rate of $\sim 20-50 / 100,000$ (1,2). Despite the specificity of the pathological location and the anatomical structure, the majority of patients are unfortunately diagnosed at advanced stage (3). Currently, radiotherapy is the mainstay of treatment for NPC. However, a high proportion of NPC patients, particularly patients with advanced NPC, exhibit radioresistance and exhibit poor outcomes $(4,5)$. Therefore, exploring the molecular mechanisms underlying sensitivity to radiation or resistance as well as the combination of chemoand radiotherapy is of crucial significance for NPC therapy.

Curcumin (diferuloylmethane, Cur) is a non-flavonoid polyphenol derived from turmeric plants that demonstrates great potential in tumorigenesis and tumor progression $(6,7)$. Accumulating evidence has indicated that Cur can sensitize numerous radioresistant tumor cells, including NPC (8-10). Our previous work also demonstrated that Cur could enhance the radiosensitivity in the CNE2 cell line at an appropriate concentration $(11,12)$. Although recent discoveries have revealed the underlying mechanisms to a certain extent, the relevance of the association between Cur and its radiosensitivity to NPC and the function of related miRNAs are not fully recognized.

MicroRNAs (miRNAs) are a class of small endogenous non-coding RNA that can regulate gene expression at the post-transcriptional level by inhibiting translation of messenger RNA and by inducing mRNA degradation (13). Recent studies indicate that various biological and pathological processes, including cellular proliferation, differentiation, and apoptosis, have been caused by the deregulation of mRNAs (14-16). Among the mRNAs, miR-593 has been reported to be down-exposed in esophageal and gastric cancer (17). The aim of the present study was to investigate whether miR-593 could radiosensitize the NPC cell line CNE2 in vitro and in vivo by regulating multidrug resistance gene 1 (MDR1), and to identify whether MDR1 is a direct and functional target of miR-593.

\section{Materials and methods}

Cell culture and reagents. The human NPC cell line CNE2 was obtained from Sun Yat-sen University (Guangzhou, China) and cultured in RMPI-1640 medium (Invitrogen; Thermo Fisher Scientific, Inc., Waltham, MA, USA) supplemented with $10 \%$ fetal bovine serum (Gibco; Thermo Fisher 
Scientific, Inc.) in a humid atmosphere containing $5 \% \mathrm{CO}_{2}$ at $37^{\circ} \mathrm{C}$. Cur (Sigma-Aldrich, St. Louis, MO, USA) was dissolved in $0.5 \%$ DMSO (Sigma-Aldrich) and diluted with RPMI-1640 medium to the desired concentrations.

Clonogenic survival assay. Cells were divided into three groups: control group (CN), IR (irradiation) group (CX), and $\mathrm{IR}+$ Cur group (JX). Cells were seeded in 6-well plates and routinely cultured for $24 \mathrm{~h}$. The CX and JX groups were radiated with X-rays at $6 \mathrm{MV}$ using $600 \mathrm{C} / \mathrm{D}$ linear accelerator (Varian Medical Systems, Inc., Palo Alto, CA, USA) to deliver the indicated doses $(2,4,6$ and $8 \mathrm{~Gy})$, and all cells were further cultured for another 12 days. The cells were fixed and stained with methanol containing 1\% crystal violet (Beijing Dingguo Changsheng Biotech Co., Ltd., Beijing, China). Colonies ( $\geq 50$ cells) were counted under the microscope (U-LH100L-3; Olympus Corporation, Tokyo, Japan) by using the following formula: Plate clone formation efficiency $=$ number of colonies/number of cells inoculated (18).

Animal studies. The study was conducted in accordance with the guideline for the Administration of Affairs Concerning Experimental Animals, and all procedures involving animals were approved by Animal Care and Ethics Committee of Southern Medical University. Balb/c nude mice (Experimental Animal Center of Southern Medical University, Weifang, China) for tumor implantation were 4-6 weeks old with a body mass of $18-22 \mathrm{~g}$. The mice were housed under controlled laboratory conditions at an ambient temperature of $23 \pm 2^{\circ} \mathrm{C}$ for 2 weeks.

For the xenograft tumor assay, $1 \times 10^{6}$ cells in $200 \mu \mathrm{l}$ of RMPI-1640 were injected subcutaneously into the right flank of nude mice. The mice were divided into 5 groups of 6 mice when the tumor volume reached $61 \mathrm{~mm}^{3}$. Cur was intragastrically administered at 3 different dosages [50, 100 (which was determined to be the optimal concentration of Cur, according to the results of a preliminary test) and $150 \mathrm{mg} / \mathrm{kg}$ ] once a day for 7 days. Saline was injected as a control. After 7 days, all groups were irradiated with 4 Gy IR every other day, 3 times. Following the final treatment, mice were euthanized, and the tumors were dissected and weighed.

Reverse transcription-quantitative polymerase chain reaction $(R T-q P C R)$ analysis. Total RNA from cells and nude mice was extracted using TRIzol reagent (Invitrogen; Thermo Fisher Scientific, Inc.), following the manufacturer's instructions. Quantification was performed using the Quantitect SYBR Green PCR Kit (Stratagene, San Diego, CA, USA) with an MX3005P multiplex quantitative qPCR system (Stratagene), according to the manufacturer's instructions. GAPDH and U6 were used as the internal control for detecting mRNA and miRNA, respectively. The comparative $\Delta \Delta \mathrm{Cq}$ method was employed as previously described (19). The fold changes were calculated according to $2^{-\Delta \Delta \mathrm{Cq}}$ equation. All of the primers used are listed in Table I.

Western blot analysis. The portion of xenograft tumors were extracted by RIPA buffer with protease inhibitors (Cell Biolabs Inc., San Diego, CA, USA) and quantified by the BCA method (Thermo Fisher Scientific, Inc.). Equal amounts of total protein
Table I. Primers used in this study.

Primer

name

Primer sequence

\begin{tabular}{ll} 
GAPDH & F: ATCATCAGCAATGCCTCCTG \\
& R: ATGGACTGTGGTCATGAGTC \\
MDR1 & F: TCATTCGAGTAGCGGCTCTT \\
& R: CTTCTTTGCTCCTCCATTGC \\
U6 & F: CTCGCTTCGGCAGCACA \\
& R: AACGCTTCACGAATTGCGT \\
miR-593 & F: TGTCTCTGCTGGGGTTCT \\
& R: GTGCAGGGTCCGAGGTATT \\
pGL3- & F: gattatagaACTCTGACTGTATGAGATGT \\
MDR1 & R: gattatagaTCACATGAAAGTTTAGT \\
si-MDR1 & S: CAGAAAGCUUAGUACCAAAdTdT \\
Si-NC & S: UAACGACGCGACGACGUAAdTdT \\
\hline
\end{tabular}

A

\begin{aligned} Position 135-141 of MDR1 3' UTR & \multicolumn{2}{c}{ 5'..CAAGUUCAGAGUCUUCAGAGACU... } \\ hsa-miR-593 & 3' \end{aligned}

Position 165-171 of MDR1 3' UTR 5 '...UAAAGGAACAGAGUGAGAGACAU.... hsa-miR-593 $\quad 33^{\prime}$ UCUUUGGGGUCGUCUCUGU

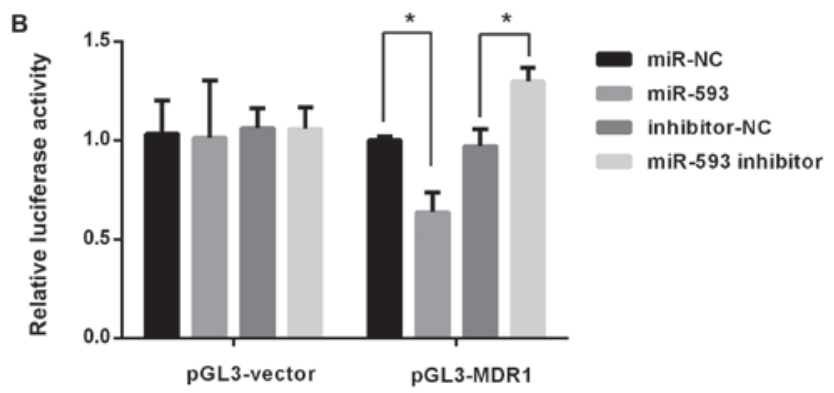

Figure 1. Effect of the putative miR-593 binding site derived from the MDR1 3'UTR on luciferase expression. (A) Schematic of the potential miR-593 binding sites containing MDR1 3'UTR. (B) Luciferase activity in HEK293 cells transfected with miR-NC, miR-593 mimics, inhibitor-NC, and miR-593 inhibitor with pGL3-vector or pGL3-MDR1 $36 \mathrm{~h}$ post-transfection. . Data represent mean \pm standard deviation of 3 independent experiments, and each experiment was performed in triplicate. ${ }^{*} \mathrm{P}<0.05$. MDR1, multidrug resistance gene 1; 3'UTR, 3'-untranslated region; miR, micro RNA.

(30-50 $\mu \mathrm{g}$ ) were resolved by $10 \%$ SDS-PAGE (Bio-Rad Laboratories, Inc., Hercules, CA, USA) and transferred onto the PVDF membrane (Thermo Fisher Scientific, Inc.), which was blocked in 5\% non-fat milk at room temperature for $1 \mathrm{~h}$. Thereafter, they were incubated with rabbit monoclonal anti-MDR1 antibody (1:2,000; catalogue no. ab170904; Abcam, Cambridge, UK) overnight at $4^{\circ} \mathrm{C}$. The membranes were blotted for $1 \mathrm{~h}$ at room temperature with goat anti-rabbit horseradish peroxidase-conjugated immunoglobulin G secondary antibody (1:1,000; catalogue no. 7074; Cell Signaling Technology, , Inc., Danvers, MA, USA). The bands were developed using ECL Kit (Cell Signaling Technology, Inc.) and quantified on Gel Logic 2200 PRO Imaging System (Kodak, Rochester, NY, USA). 

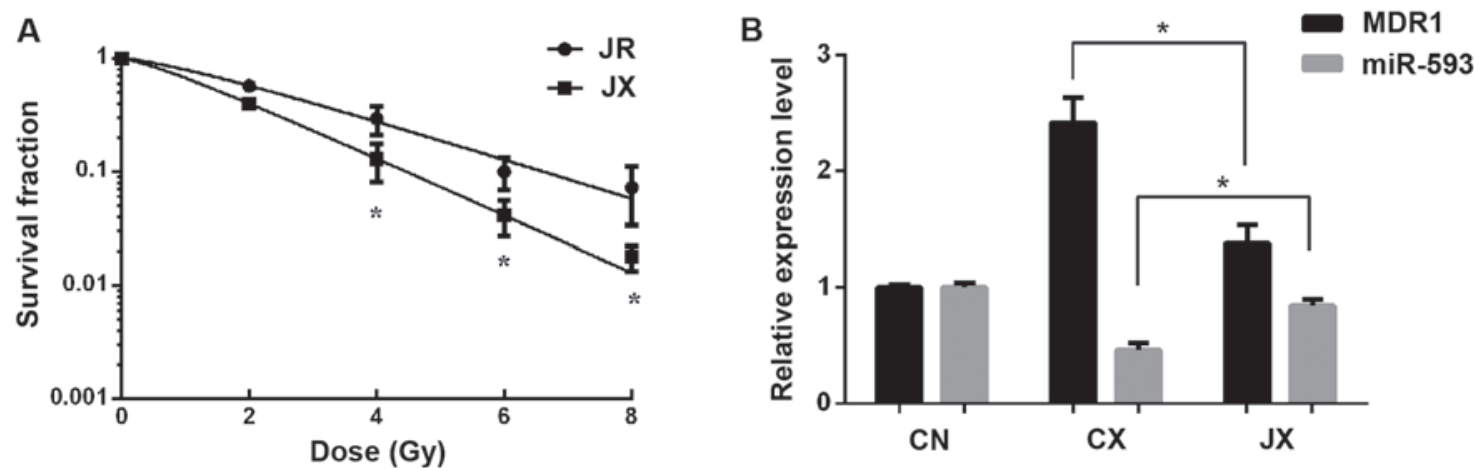

Figure 2. Cur sensitizes nasopharyngeal carcinoma cells to irradiation treatment through upregulating mir-593 to reverse IR induced MDR1 expression. (A) CNE2 cells were treated with $0,2,4,6$, or 8 Gy of IR with Cur pretreatment. The cell survival fraction was calculated by clonogenic assay. (B) Relative miR-593 and MDR1 expression were determined by quantitative polymerase chain reaction. IR-induced miR-593 downregulation and MDR1 upregulation were reversed during Cur-enhanced radiosensitization. Data are presented as mean \pm standard deviation from 3 replicate experiments. "P $<0.05$. CN, control group; CX,irradiated group; IX, irradiation +Cur group; Cur, curcumin; MDR1, multidrug resistance gene 1; IR, irradiation; miR, micro RNA.
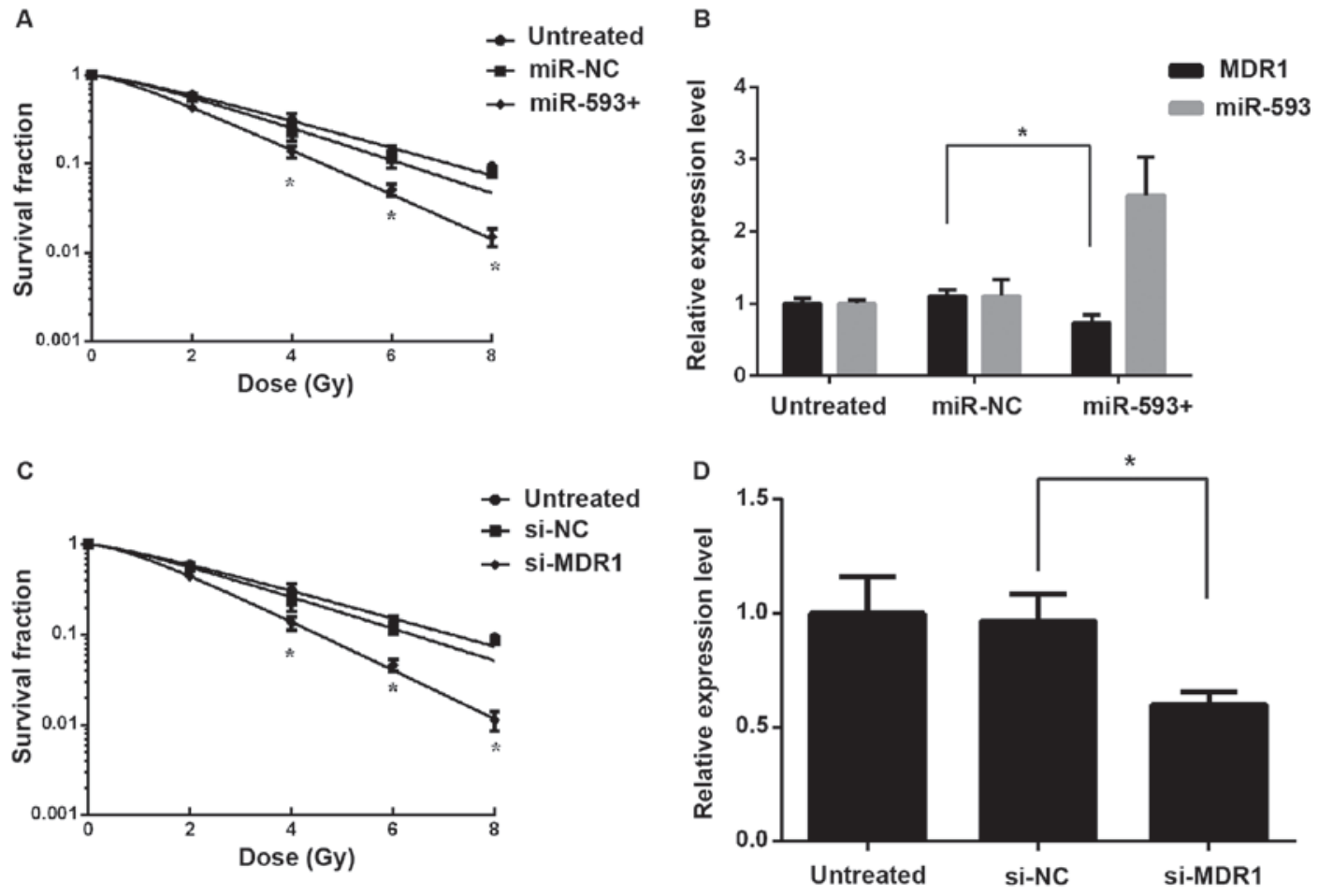

Figure 3. Effect of MDR1 knockdown on radiosensitivity of nasopharyngeal carcinoma cells in vitro. (A) Survival fraction was calculated by clonogenic assay after treatment with miR-593 mimics (miR-593+) and (B) relative miR-593 and MDR1 expression were determined by qPCR. (C) Survival fraction after treatment with si-MDR1 and (D) relative miR-593 and MDR1 expression were determined by qPCR. All data are presented as mean \pm standard deviation from 3 replicate experiments. "P<0.05. Cur, curcumin; MDR1, multidrug resistance gene 1; si, short interfering; qPCR, quantitative polymerase chain reaction; miR, micro RNA.

Luciferase assay. The entire 380-base pair fragment of MDR1 3' UTR that contains the putative binding site of miR-593 was amplified by PCR and cloned downstream of the luciferase gene at the XbaI sites in the pGL-3 plasmid (Promega Corporation, Madison, WI, USA). The construct was designated as pGL3-MDR1 (primers are listed in Table I). HEK293 cells were co-transfected with $30 \mathrm{pmol}$ of either miR-593 mimics or miR-593 NC and pGL3-MDR1 using Lipofectamine 2000 (Invitrogen; Thermo Fisher Scientific, Inc.). Luciferase activity was measured using the Promega dual-luciferase assay kit and normalized by $\beta$-galactosidase activity. Relative protein levels were expressed as Renilla/firefly luciferase ratios. Each experiment was repeated twice (11).
RNA interference. CNE2 cells at $20-30 \%$ confluence were transfected with $50 \mathrm{nM}$ of siRNAs using Lipofectamine 2000 following the manufacturer's protocol. Small interfering RNA (siRNA) and scrambled negative control siRNA (siRNA-NC) were obtained from Invitrogen (Thermo Fisher Scientific, Inc.). The target sequence of MDR1 is listed in Table I. A total of $36 \mathrm{~h}$ after transfection, cells were harvested for RT-qPCR.

Statistics. SPSS software, version 13.0 (SPSS Inc., Chicago, IL, USA) was used for all the statistical analyses in the present study. Quantitative data are presented as mean \pm standard deviation. $\chi^{2}$ test and Student's t-test were appropriately applied 
A

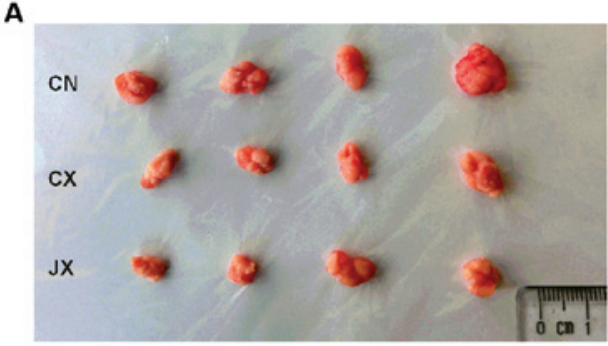

C

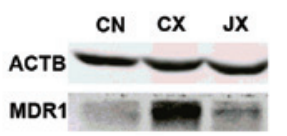

B

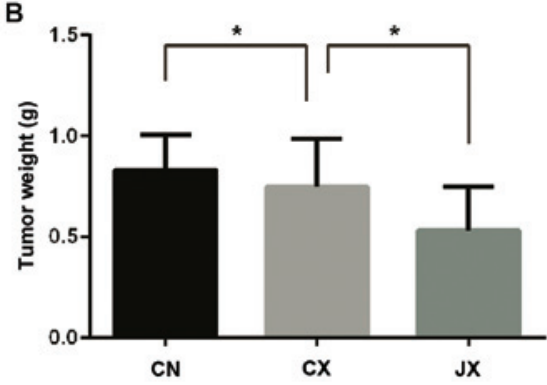

D

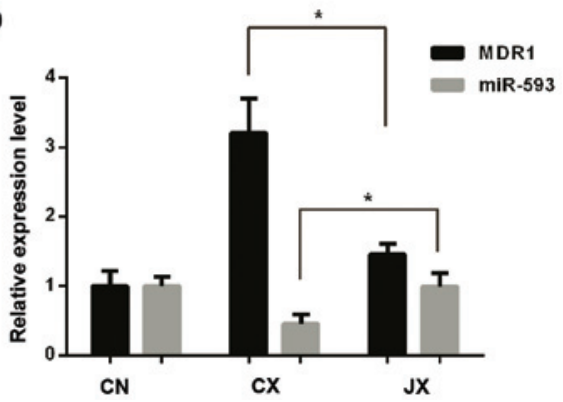

Figure 4. Radiosensitization of Cur in xenograft tumors in vivo. (A) Image of tumors in each group. (B) Tumor weight of each group. (C) Relative mir-593 and MDR1 expression were determined by (C) western blot assay or (D) qPCR. All data are presented as mean \pm standard deviation, ${ }^{*} \mathrm{P}<0.05$. Cur, curcumin; MDR1, multidrug resistance gene 1 ; qPCR, quantitative polymerase chain reaction; miR, micro RNA; CN, control group; CX,irradiated group; IX, irradiation +Cur group.

for different types of data. $\mathrm{P}<0.05$ was considered to indicate a statistically significant difference.

\section{Results}

MDRl is a direct target of miR-593. A bioinformatics analysis was performed using TargetScan version 7.0 software (http:// www.targetscan.org/), which predicted that miR-593 may target the MDR1 3'UTR region (Fig. 1A). A luciferase-based reporter was constructed to evaluate the effect of miR-593 direct binding to the putative target site on the 3'UTR of MDR1. To substantiate the assumption that miR-593 can directly repress MDR1, the reporter-construct pGL3-vector and pGL3-MDR1 were co-transfected with miR-593 mimic, miR-NC, and miR-593 inhibitor or inhibitor-NC to HEK293 cells. Luciferase activity was then assayed. As shown in Fig. 1B, for pGL3-MDR1 construct, miR-593 mimic significantly lowered luciferase activity compared with miR-NC $(\mathrm{P}<0.05)$. By contrast, miR-593 inhibitor increased luciferase activity in HEK293 cells compared with inhibitor-NC $(\mathrm{P}<0.05)$. These findings support the hypothesis that miR-593 directly targets MDR1 expression.

Cur sensitizes CNE2 cells to IR through upregulating mir-593 to reverse $I R$-induced MDRl expression. The colony survival assay is considered a canonical standard to determine radiosensitivity (20). The results confirmed that cells pretreated with Cur were much more sensitive to IR than their untreated counterparts. The $\alpha$ and $\beta$ components were $0.2476 / \mathrm{Gy}$ and $0.01650 / \mathrm{Gy}^{2}$ for the cells treated with radiation alone, and $0.4201 / \mathrm{Gy}$ and $0.02029 / \mathrm{Gy}^{2}$ for the cells treated with the combination treatment (Fig. 2A), respectively, leading to significantly different $(\mathrm{P}<0.001)$ survival fractions, as tested with the linear regression analysis. The data were further analyzed according to the multitarget single-hit model. When pretreated with $10 \mu \mathrm{M}$ Cur $\left(\mathrm{IC}_{20}\right)$, the sensitization enhancement ratio of
CNE-2 reached 1.44. These data indicate that Cur has an effective radiosensitization effect on the CNE-2 cell line in vitro. In addition, miR-593 and MDR1 expression were detected by RT-qPCR (Fig. 2B). The result demonstrated that IR-induced miR-593 downregulation was reversed during Cur-enhanced radiosensitization $(\mathrm{P}<0.05)$.

The effect of MDRl knockdown on the radiosensitivity of NPC cells in vitro. Cur enhances the radiosensitivity involving the reversal of differentially expressed mir-593 and MDR1. To elucidate whether the effect of Cur on radiosensitivity was mediated by repression of MDR1, mir-593 mimics or si-MDR1 were transfected into CNE2 cells. A clonogenic assay suggested that the ectopic expression of MDR1 significantly reduced miR-593-induced radiosensitivity (Fig. 3A and C), which was consistent with the results of RT-qPCR (Fig. 3B and D). These observations indicated that Cur may have sensitized cells to IR by stimulating mir-593 to downregulate the expression of MDR1.

Radiosensitization of Cur in xenograft tumors in vivo. The tumor weight of each mouse in each treatment group was measured (Fig. 4A). A single dose of 4 Gy irradiation (CX group) exhibited the expected effect of tumor growth inhibition compared with the untreated $\mathrm{CN}$ group $(\mathrm{P}<0.05$, Fig. $4 \mathrm{~B})$. However, the JX group (Cur $100 \mathrm{mg} / \mathrm{kg}$ and 4 Gy irradiation) had the highest inhibition ratio $(62.18 \%)$ and expressed a significant tumor growth inhibition effect compared with the CX group $(\mathrm{P}<0.05$, Fig. 4B). Mice body weights were monitored to assess the tolerability of systemic Cur. Body weight changes over the course of the experiment were minimal in all treatment groups, suggesting that Cur is well tolerated (data not shown).

The expression level of miR-593 was significantly higher in the JX group compared with the CX group. To investigate the regulation of MDR1 by miR-593, the relative expression of MDR1 was measured by western blot assay (Fig. 4C) and 
RT-qPCR (Fig. 4D). In accordance with the altered expression level of miR-593, MDR1 was significantly downregulated in the JX group. These findings also indicate that MDR1 is a target of miR-593.

\section{Discussion}

Radiotherapy is considered one of the most effective treatments for patients with NPC, and radioresistance is the main risk factor that contributes to poor prognosis (21). Radioresistance occurs in primary IR treatment, and the survived cells may be more resistant to the second IR treatment, thereby leading to radiotherapy failure $(12,22,23)$. In this regard, the exact molecules and signaling pathway involved in radiosensitization should be determined to develop target therapy and enhance the efficacy of radiation. In the present study, it was observed that IR-induced downregulation of miR-593 or upregulation of MDR1 expression was almost reversed by Cur.

Cur regulates the gene expression involved in survival, proliferation, angiogenesis, invasion, and metastasis. This phytochemical also modulates various mechanisms that are associated with radioresistance, including the following: downregulating COX-2, MRP, Bcl-2, and survivin expression; inhibiting PI3K/AKT activation; suppressing growth factor signaling pathways; and inhibiting STAT3 activation (24-26). The present study demonstrated that Cur enhanced radiosensitivity in the NPC cell line CNE2 at $10 \mu \mathrm{mol} / 1$ by MTT or clonogenic survival test (27), although Cur exhibited higher anti-proliferative effects when used alone at a concentration of 20 or $40 \mu \mathrm{mol} / 1$. Considering the cytotoxicity of Cur and IR, a concentration of $10 \mu \mathrm{mol} / 1$ and $24 \mathrm{~h}$ pretreatment was more suitable as a radioenhancer (data not shown). In addition, in the animal studies, Cur $100 \mathrm{mg} / \mathrm{kg}$ was chosen as optimal concentration after a preliminary test. Therefore, no significant data were obtained by conjoint analysis with other groups although the single Cur group (JN group) was performed (data not shown). In the present study, the radiosensitizing effect of Cur was evaluated in vitro and in vivo. The data indicated that the radiosensitizing effect of Cur may be associated with mir-593 and MDR1.

P-glycoprotein (P-gp), encoded by MDR1, has attracted great interest because of its role in MDR in a variety of cancers. P-gp is overexpressed in cancer cells that actively extrude chemotherapeutic agents (28). MDR1 mediates not only chemoprotection by drug efflux but has also been found to inhibit apoptosis induced by chemotherapeutics, death receptor ligands, serum starvation, and UV or ionizing irradiation. Therefore, blocked MDR1 may enhance the efficacy of chemotherapy and reverse radioresistance in patients. Maier et al (29) confirmed that a protective effect of retroviral overexpression of MDR1 is increasing the radiotolerance of haematopoietic cells and the related apoptosis gene was downregulated. In the present study, it was also observed that MDR1 was a radioresistant factor which could be downregulated by mir-593.

Certain miRNAs were reported to affect the radiosensitivity of cancer cells, such as let-7, miR-21, miR-101, miR-421, and miR-181a (30-34). In esophageal cancer (EC), miR-593 degrades polo-like kinase 1 (PLK1) mRNA by direct binding to the 3'-UTR of PLK1 mRNA and reduced proliferation of
EC cells (17). Bioinformatics analysis was used in the present study to predict that miR-593 may also target the MDR1 3'UTR region. A luciferase-based reporter showed that miR-593 regulated MDR1 expression by directly targeting 3'UTR, consistently resulting in reduced expression of MDR1. Furthermore, modulated expression tests demonstrated that radiosensitization of Cur was triggered by miR-593 instead of directly by MDR1.

Taken together, the results demonstrated that Cur had a radiosensitization effect on NPC cells in vivo and in vitro; curcumin-mediated upregulation of miR-593 causes the depression of MDR1 expression, which may promote radiosensitivity of NPC cells.

\section{Acknowledgements}

The present study was supported by the National Natural Science Foundation of China (grant nos. 81173616, 81202430 and 81302948) and Guangdong Science and Technology Project (grant no. 2013A032500003), and Science and Technology Project of Haizhu District (grant no. 2013-cg-29); the authors thank medical personnel Mr. Jiabin Liu and Ms. Huarui Niu of NanFang Hospital for providing X-ray radiation equipment.

\section{References}

1. Loong HH, Ma BB and Chan AT: Update on the management and therapeutic monitoring of advanced nasopharyngeal cancer. Hematol Oncol Clin North Am 22: 1267-1278, 2008.

2. Chan AT, Teo PM and Johnson PJ: Nasopharyngeal carcinoma. Ann Oncol 13: 1007-1015, 2002.

3. Jemal A, Bray F, Center MM, Ferlay J, Ward E and Forman D: Global cancer statistics. CA Cancer J Clin 61: 69-90, 2011.

4. Sanguineti G, Geara FB, Garden AS, Tucker SL, Ang KK, Morrison WH and Peters LJ: Carcinoma of the nasopharynx treated by radiotherapy alone: Determinants of local and regional control. Int J Radiat Oncol Biol Phys 37: 985-996, 1997.

5. Siegel R, Naishadham D and Jemal A: Cancer statistics, 2013. CA Cancer J Clin 63: 11-30, 2013.

6. Ji JL, Huang XF and Zhu HL: Curcumin and its formulations: Potential anti-cancer agents. Anticancer Agents Med Chem 12: 210-218, 2012.

7. Rahmani AH, AlZohairy MA, Aly SM and Khan MA: Curcumin: A potential candidate in prevention of cancer via modulation of molecular pathways. Biomed Res Int 2014: 761608, 2014.

8. López-Jornet P, Camacho-Alonso F and Gómez-Garcia F: Effect of curcumin and irradiation in PE/CA-PJ15 oral squamous cell carcinoma. Acta Odontol Scand 69: 269-273, 2011.

9. Chang YJ, Huang CY, Hung CS, Chen WY and Wei PL: GRP78 mediates the therapeutic efficacy of curcumin on colon cancer. Tumour Biol 36: 633-641, 2015.

10. Xie YQ, Wu XB and Tang SQ: Curcumin treatment alters ERK-1/2 signaling in vitro and inhibits nasopharyngeal carcinoma proliferation in mouse xenografts. Int J Clin Exp Med 7: 108-114, 2014

11. Liu Y, Cai H, Liu J, Fan H, Wang Z, Wang Q, Shao M, Sun X, Diao J, Liu Y, et al: A miR-151 binding site polymorphism in the 3'-untranslated region of the cyclin E1 gene associated with nasopharyngeal carcinoma. Biochem Biophys Res Commun 432: 660-665, 2013

12. Wang Q, Fan H, Liu Y, Yin Z, Cai H, Liu J, Wang Z, Shao M, Sun X, Diao J, et al: Curcumin enhances the radiosensitivity in nasopharyngeal carcinoma cells involving the reversal of differentially expressed long non-coding RNAs. Int J Oncol 44: 858-864, 2014.

13. Djuranovic S, Nahvi A and Green R: A parsimonious model for gene regulation by miRNAs. Science 331: 550-553, 2011.

14. Yang B, Jing C, Wang J, Guo X, Chen Y, Xu R, Peng L, Liu J and $\mathrm{Li}$ L: Identification of microRNAs associated with lymphangiogenesis in human gastric cancer. Clin Transl Oncol 16: 374-379, 2014. 
15. Zhang T, Sun Q, Liu T, Chen J, Du S, Ren C, Liao G and Yuan Y: MiR-451 increases radiosensitivity of nasopharyngeal carcinoma cells by targeting ras-related protein 14 (RAB14). Tumour Biol 35: 12593-12599, 2014.

16. Nygren MK, Tekle C, Ingebrigtsen VA, Mäkelä R, Krohn M, Aure MR, Nunes-Xavier CE, Perälä M, Tramm T, Alsner J, et al: Identifying microRNAs regulating B7-H3 in breast cancer: The clinical impact of microRNA-29c. Br J Cancer 110: 2072-2080, 2014.

17. Ito T, Sato F, Kan T, Cheng Y, David S, Agarwal R, Paun BC, Jin Z, Olaru AV, Hamilton JP, et al: Polo-like kinase 1 regulates cell proliferation and is targeted by miR-593* in esophageal cancer. Int J Cancer 129: 2134-2146, 2011.

18. Zhang Y, Zheng L, Huang J, Gao F, Lin X, He L, Li D, Li Z, Ding Y and Chen L: MiR-124 Radiosensitizes human colorectal cancer cells by targeting PRRX1. PLoS One 9: e93917, 2014.

19. Livak KJ and Schmittgen TD: Analysis of relative gene expression data using real-time quantitative PCR and the $2^{-\Delta \Delta C T}$ method. Methods 25: 402-408, 2001.

20. Yaromina A, Krause M, Thames H, Rosner A, Krause M, Hessel F, Grenman R, Zips D and Baumann M: Pre-treatment number of clonogenic cells and their radiosensitivity are major determinants of local tumour control after fractionated irradiation. Radiother Oncol 83: 304-310, 2007.

21. Lu ZX, Ma XQ, Yang LF, Wang ZL, Zeng L, Li ZJ, Li XN, Tang M, Yi W, Gong JP, et al: DNAzymes targeted to EBV-encoded latent membrane protein-1 induce apoptosis and enhance radiosensitivity in nasopharyngeal carcinoma. Cancer Lett 265: 226-238, 2008.

22. Pearce AG, Segura TM, Rintala AC, Rintala-Maki ND and Lee H: The generation and characterization of a radiation-resistant model system to study radioresistance in human breast cancer cells. Radiat Res 156: 739-750, 2001.

23. Baldwin AS: Control of oncogenesis and cancer therapy resistance by the transcription factor NF-kappaB. J Clin Invest 107: 241-246, 2001.

24. Kunnumakkara AB, Diagaradjane P, Guha S, Deorukhkar A, Shentu S, Aggarwal BB and Krishnan S: Curcumin sensitizes human colorectal cancer xenografts in nude mice to gamma-radiation by targeting nuclear factor-kappaB-regulated gene products. Clin Cancer Res 14: 2128-2136, 2008.
25. Javvadi P, Segan AT, Tuttle SW and Koumenis C: The chemopreventive agent curcumin is a potent radiosensitizer of human cervical tumor cells via increased reactive oxygen species production and over activation of the mitogen-activated protein kinase pathway. Mol Pharmacol 73: 1491-1501, 2008.

26. Narang $\mathrm{H}$ and Krishna $\mathrm{M}$ : Inhibition of radiation induced nitration by curcumin and nicotinamide in mouse macrophages. Mol Cell Biochem 276: 7-13, 2005.

27. Hannoun-Levi JM, Chand-Fouche ME, Dejean C and Courdi A: Dose gradient impact on equivalent dose at 2 Gy for high dose rate interstitial brachytherapy. J Contemp Brachytherapy 4: 14-20, 2012.

28. Montazami N, Aghapour M, Farajnia S and Baradaran B: New insights into the mechanisms of multidrug resistance in cancers. Cell Mol Biol (Noisy-le-grand) 61:70-80, 2015.

29. Maier P, Herskind C, Fleckenstein K, Spier I, Laufs S, Zeller WJ, Fruehauf S and Wenz F: MDR1 gene transfer using a lentiviral SIN vector confers radioprotection to human CD34+ hematopoietic progenitor cells. Radiat Res 169: 301-310, 2008.

30. Oh JS, Kim JJ, Byun JY and Kim IA: Lin28-let7 modulates radiosensitivity of human cancer cells with activation of K-Ras. Int J Radiat Oncol Biol Phys 76: 5-8, 2010.

31. van Jaarsveld MT, Wouters MD, Boersma AW, Smid M, van Ijcken WF, Mathijssen RH, Hoeijmakers JH, Martens JW, van Laere $\mathrm{S}$, Wiemer EA and Pothof J: DNA damage responsive microRNAs misexpressed in human cancer modulate therapy sensitivity. Mol Oncol 8: 458-468, 2014.

32. Sun Q, Liu T, Zhang T, Du S, Xie GX, Lin X, Chen L and Yuan Y: MiR-101 sensitizes human nasopharyngeal carcinoma cells to radiation by targeting stathmin 1 . Mol Med Rep 11: 3330-3336, 2015.

33. Mansour WY, Bogdanova NV, Kasten-Pisula U, Rieckmann T, Köcher S, Borgmann K, Baumann M, Krause M, Petersen C, $\mathrm{Hu} \mathrm{H}$, et al: Aberrant overexpression of miR-421 downregulates ATM and leads to a pronounced DSB repair defect and clinical hypersensitivity in SKX squamous cell carcinoma. Radiother Oncol 106: 147-154, 2013.

34. Ke G, Liang L, Yang JM, Huang X, Han D, Huang S, Zhao Y, Zha R, He X and Wu X: MiR-181a confers resistance of cervical cancer to radiation therapy through targeting the pro-apoptotic PRKCD gene. Oncogene 32: 3019-3027, 2013. 